\title{
LA OMNIPRESENTE SELECCIÓN NATURAL
}

\section{THE OVERALL PRESENCE OF NATURAL SELECTION}

\author{
Andrés GALERA \\ GEA, Instituto de Historia, CSIC*
}

\begin{abstract}
RESUMEN: El concepto de selección natural da título y sirve de pretexto para reflexionar sobre el modelo de naturaleza representado por el principio biológico de evolución: desde Lamarck en adelante con parada en el giro darwiniano que mediado el siglo modela la idea en clave selectiva. La naturaleza está así sometida al azaroso principio de sobrevivir por competencia obligada (selección natural), definiendo la adaptación al medio como un proceso fortuito frente a la necesaria adaptación teleológica propuesta por el adversario. La historia evolutiva puede reconstruirse como una hilera de cuentas pendientes, de continuadas disputas entre defensores y detractores de la selección natural, explicando cómo y por qué la naturaleza cambia cronológicamente hacia formas alternativas.
\end{abstract}

PalabRas ClaVE: Darwin, evolución, Lamarck, Mendel, selección natural, guerra natural.

ABSTRACT: Natural selection is used as title and argument to think about the model of nature represented by the biological principle of evolution: from Lamarck forward on with a stop in the Darwinian option that in middle century shapes the underlying idea in a selective key. Nature is submitted to the fortuitous principle of surviving under forced competition (natural selection), defining adaptation to the environment as indeed a fortuitous process opposite to a

* Proyecto de investigación n. ${ }^{\circ}$ HAR2009-12418. 
necessary teleological adjustment proposed by the adversary. Evolutionary history can be reconstructed as a collection of continued revenges between defender and supporter of natural selection, explaining how and why nature transforms itself in the course of time towards alternative forms.

KEYWORDS: Darwin, evolution, Lamarck, Mendel, natural selection, nature war.

Pasaron los años, doscientos, desde que Jean-Baptiste Lamarck publicó su libro Philosophie zoologique, un tratado materialista relativo a la vida animal tanto en su vertiente morfológico-organizativa como a nivel fenomenológico-funcional, contenedor de dos conceptos epistemológicos básicos para la interpretación moderna de la naturaleza: uno, el común origen biológico de los seres vivos a partir de materia y por la acción conjunta de procesos físico-químicos comunes, generándose propiedades animadas y sensoriales genuinas; dos, el principio transformista por el cual los organismos se modifican cronológicamente en función de sus necesidades adaptativas para sobrevivir en nuevos ambientes. Lamarck lo piensa y Niels Bohr lo escribe en los años 30 del siglo XX, la vida es el postulado fundamental de la biología ${ }^{1}$; fundacional diríamos. El individuo la representa, plasma el proceso diferenciador de la materia, y ese colectivo llamado especie idealiza una convergencia formal válida sólo mientras permanezcan las condiciones del medio ${ }^{2}$. La doctrina lamarckiana supone una renovación del pensar naturalista con rumbo decidido hacia la biología moderna, y no por casualidad. Realmente, su proyecto era escribir una monografía titulada Biología pero se conformará con dar rienda suelta a su pensamiento respetando la clásica fórmula nominal de Filosofía ${ }^{3}$, que no hace justicia al innovador contenido.

Cuando el año 1859 se publica $O n$ the origin of species by means of natural selection hace cincuenta años que el ideario transformista lamarckiano circula por

1 Citamos por BoHr, N., I quanti e la vita, unità de la natura unità della conoscenza, Bollati, Turín, 1999, pp. 45-46.

2 Cf. Galera, A., "Lamarck y la conservación adaptativa de la vida", Asclepio, vol. LXI, 2, 2009, pp. 129-140.

3 Cf. Lamarck, J. B., Philosophie zoologique (Avertissement), Flammarion, París, 1994, p. 61. 
el mundillo científico ${ }^{4}$ proyectando la imagen atípica de un mundo orgánico caracterizada por su variabilidad; esquema rechazado por algunos, ignorado por bastantes y compartido por los demás, incluido Charles Darwin quien, mucho antes de la célebre añada del 59, en enero de 1844, reconoce haber llegado a conclusiones similares a las formuladas por Lamarck sobre la transformación de los seres vivos ${ }^{5}$. Básicamente, su discrepancia radica en el mecanismo productor del cambio. La peculiaridad del testimonio darwiniano estriba en subrayar el consenso entorno a la idea lamarckiana de interpretar la vida como un continuado proceso orgánico sustitutivo de unas especies por otras más adaptadas; que la adaptación sea teleológica o azarosa en mayor o menor medida diferencia la teoría de cada cual en función de la solución adaptativa aportada. Acudimos al paleontólogo Geoge Gaylord Sympson para suscribir que Lamarck recurrió valientemente a la evolución "como explicación general de la historia de la vida»; siendo capaz de articular un armazón cognitivo entorno al ser vivo convertido en la base ideológica de la teoría evolutiva. Sintonizando esta frecuencia, la darwiniana evolución por selección natural expuesta en el Origen se presenta como alternativa modélica que no conceptual; la selección competitiva es una receta metodológica para resolver parcialmente el reto cognitivo, el problema biológico-evolutivo planteado por Lamarck: cómo y porqué cambian las especies durante la historia terrestre. El antievolucionista Armand de Quatrefages lo tuvo claro hace más de un siglo. Despojada de la selección natural, el componente intrínseco, la doctrina de Darwin se reduce a la conocida idea transformista ${ }^{7}$; nada nuevo bajo el Sol. Un biempensante de la causa darwinista como Julian Huxley convino en el análisis declamando que la propuesta de Darwin no es la idea de evolución sino el mecanismo que la explica razonablemente ${ }^{8}$. El modelo evolutivo por transfor-

4 Cf. Laurent, G., La naissance du transformismo.Lamarck entre Linné et Darwin, Vuibert, París, 2001. También GalerA, A., «Modelos evolutivos predarwinistas», Arbor, 172 (677), 2002, pp. 1-16. GALERA, A., «La alquimia de la vida. Etienne Geoffroy Saint-Hilaire y el evolucionismo experimental; en Guedes, E. (ed.), Numeros e outras coisas da vida, Apenas livros, Lisboa, 2006, pp. 3-18.

5 Carta de Ch. Darwin a J. Hooker, 11 de enero de 1844, en DARWIN, F. (ed.), The Autobiography of Charles Darwin and Selected letters, Dover, Nueva York, 1958, p.184 (1. a ed. 1892).

${ }^{6}$ Simpson, G. G., La vida en el pasado, Madrid, Alianza, 1967, p. 186 (Life of the past. An introduction to paleontology).

7 Quatrefages, A. De, Charles Darwin et ses précurseurs françaises, París, 1892, p.11 (1ª ed. 1870).

8 Huxley, J., "La revanche du darwinisme», conferencia impartida en el Palais de la Dècouverte, París 3 de octubre de 1945; texto en Drouin, J-M.; LenAY, CH. (eds,), Théories de l'évolution, une anthologie, Pocket, París, 1990, p.162. 
mación cronológica específica se definió en la década de $1800^{9}$ y el mérito de su denominación moderna como Théorie de l'evolution des formes organiques le corresponde al botánico francés Frédéric Gérard ${ }^{10}$ alcanzados los años 40, fórmula que Darwin conoció ${ }^{11}$. Antes y después de publicarse el Origen el debate científico sobre la evolución mantuvo dos frentes abiertos; son la polémica entorno a la aceptación de la teoría, y, sólo para suscriptores, la discusión alrededor de la causa y el mecanismo que determinan el proceso. Este, y no otro, es el escenario, abierto, actual, continuo, convulso, crítico, plural, renovador, donde se incorpora, toma forma y será examinada la teoría darwiniana del origen de las especies por selección natural; nada de revolución científica al estilo kuhniano.

Es opinión manifestada por el botánico Alphonse de Candolle que el sistema propuesto por su contemporáneo Charles Darwin era el más moderno, ingenioso y completo de los formulados entonces; tal esquema selectivo-competitivo ofrecía las mayores probabilidades de éxito al explicar los datos paleontológicos, biogeográficos, anatómicos, sistemáticos, relativos al decurso temporal de los seres vivos. Darwin habría puesto el dedo en la llaga al proponer la selección natural como modo de fijar las variaciones generación tras generación. El planteamiento suponía un cambio de rumbo en la mentalidad evolucionista, aunque no lo explicaba todo y las pruebas directas resultaban insuficientes. Déficit probatorio que hacía pensar si Darwin no exageraba atribuyendo todo el protagonismo a la selección natural, descuidando otras causas posibles ${ }^{12}$. Notoriamente exultante, acérrimo, confiado en la tesis, el incondicional Thomas Huxley relata que el Origen trajo consigo «la hipótesis de trabajo que buscábamos». Un rayo en la noche oscura iluminó súbitamente el camino de la evolución en los términos de lucha por la existencia y supervivencia del más apto. La cuestión era sen-

9 Philosophie zoologique culmina el ideario transformista lamarckiano pero la idea está explícitamente presente en sus trabajos desde 1800. Cf. GALERA, A., «Lamarck y la conservación adaptativa de la vida", Asclepio, vol. LXI, 2, 2009.

10 GÉRARD, F., Dictionnaire Universel d'Histoire Naturelle: «Dégénérescence», 1844, t. 4, pp. 647-655; «Espèce», 1844, t. 5, pp. 428-452; «Géographie zoologique», 1845, t. 6, pp. 112-192; «Generation spontaneée ou primitive», 1845, t. 6, pp. 53-71. Cf. GALERA, A., «Modelos evolutivos predarwinistas", Arbor, 172 (677), 2002.

${ }_{11}$ Cf. la correspondencia de Ch. Darwin a J. D. Hooker, de fecha 19 marzo y [15 ó 22 de agosto] de [1845] (reproducida en wwww.darwinproject.ac.uk/darwinletters).

${ }^{12}$ Cf. Candolle, A. De, «Etude sur l'espèce à l'occasion d'une révision de la famille des cupuliferes», Annales des sciences naturelles, $4 .^{a}$ serie, Botanique, t. XVIII, 1862, pp.101, 103, 105. 
cilla y evidente, tanto que se consideraba un estúpido ${ }^{13}$ por su ceguera mental. Huxley padre tendría sus razones para usar el calificativo, no le desmentiremos, pero lo conveniente ahora es subrayar la convergencia de los testimonios resaltando como hecho diferenciador del acto darwiniano no la idea conocida de evolución sino el sui géneris mecanismo propuesto, la hipótesis de trabajo: la selección natural, asimilada como simplificación extrema de un texto que en su primera edición alcanzó las quinientas páginas. Darwin habría descubierto la piedra filosofal de la evolución e, intencionadamente o no, la selección identificará su doctrina. Un concepto fácil de aprender, comprender y difundir, sin necesidad de ser una lumbrera; concepto convertido en singular y oportuna abreviación de la teoría. Lo advierte Ronald Fisher en el prefacio de su conocido trabajo The genetical theory of natural selection del año 1930, cuya frase inicial es lapidaria: «Natural Selection is not Evolution» ${ }^{14}$. Escribir que la selección natural no es la evolución tampoco es una renuncia ideológica, es poner el punto sobre la í respecto al excesivo valor atribuido a la competencia como parámetro evolutivo, incluso para la hueste neodarwinista. Afirmándolo Fisher considera la selección natural como un mero componente del entramado evolutivo. Conceptualización que una década después Julian Huxley, en su Modern synthesis, etiqueta como heterogeneidad de la evolución, y bajo este epígrafe leemos que la selección natural «por sí sola es incapaz de extender el límite máximo de la variación, y, por tanto, incapaz por sí misma, de causar cambios evolutivos», en consecuencia la evolución es el producto combinado de la mutación, la recombinación y la selección ${ }^{15}$; procesos complementarios encadenados.

El mismo Darwin puso puertas al campo reconociendo este límite operativo, aunque esperó la publicación de la cuarta edición del Origen para aclarar el malentendido. «En el sentido literal de la expresión, selección natural es un nombre erróneo, no hay duda» ${ }^{16}$, dice el párrafo añadido a la entrega del 66. La selección natu-

${ }^{13}$ Cf. Drouin, J-M.; LenaY, CH. (eds,), Théories de l'évolution, une anthologie, Pocket, París, 1990, p.162.

${ }_{14}$ FISHER, R. A., The genetical theory of natural selection, Dover, Nueva York, 1958, preface, p.vii (1 ${ }^{\text {a }}$ ed. Oxford University Press, 1930).

${ }^{15}$ HuXley, J., La evolución. Sintesis moderna, Losada, Buenos Aires, 1965, 2. a ed., p. 29 (Evolution. The Modern Synthesis, G. Allen \& Unwin Ltd., Londres, 1943).

${ }^{16} C f$. DARWIN, Ch., On the origin of species by means of natural selection, John Murray, Londres, $1866,4^{\text {th }}$, p. 92 . En la 5.a y 6. ${ }^{\text {a }}$ ediciones (pp. 93 y 63) Darwin sustituye el vocablo misnome por false term. 
ral no produce variabilidad, sólo preserva las variaciones beneficiosas para el organismo en función de las condiciones de vida ${ }^{17}$. He aquí el quid de la cuestión. El modelo por la selección natural es una hipótesis restringida a explicar la presión fenotípica sin nada que decir sobre el cómo y el porqué de la variación orgánica que alimentan la evolución, puntos cruciales para interpretarla. Considerando que toda teoría evolutiva debe justificar dos niveles cognitivos básicos: primero, el vínculo genésico de las actuales formas vivas con sus antecesores; segundo, la existencia de mecanismos biológicos capaces de generar dicha variabilidad; aceptando que antes la paleontología, la embriología, la anatomía comparada, después también la genética, la bioquímica, la biología molecular, verbigracia, aportan las pruebas de primer nivel -correspondiente a la definición de evolución como principio biológico general-, es en el segundo estadio donde cada ideario particulariza la teoría. En este capítulo Darwin, contemplando el origen de las especies bajo el prisma dominante de la selección natural, asume un déficit científico invalidante relativo a la condición diversificadora de la naturaleza, sin la cual el proceso no existe. La situación mejoró el año 1868 al publicar The variation of plants and aimals under domestication ${ }^{18}$ que incluía la pangenesis theory, un mecanismo hereditario complemento de su planteamiento evolutivo. Era «una dificultad que siempre me había inquietado», escribía Alfred Russel Wallace tras conocer la buena nueva ${ }^{19}$; aunque la tranquilidad duró poco y hubo de reconocer que por su complejidad y dificultad la pangénesis mereció un rechazo generalizado ${ }^{20}$.

En idéntica dirección, la de resolver el problema del origen biológico de la biodiversidad, remaba entonces Gregor Mendel investigando el fenómeno de la hibridación botánica. El resultado de su consabida experimentación con guisantes se publicó el año 1866, información que Darwin no pudo, no quiso, no supo, no alcanzó a comprender ${ }^{21}$. La hibridación fue un obstáculo superlativo

17 Ibídem, p. 91.

18 DARWIN, Ch., The variation of plants and aimals under domestication, John Murray, Londres, 1868.

19 Carta de Wallace a Darwin, febrero 1868; cf. DARWIN, F. (ed.), The Autobiography of Charles Darwin and Selected letters, Dover, Nueva York, 1958, p. 281.

20 Wallace, A. R., Darwinism. An exposition of the theory of natural selection with some of its applications, Mcmillan, Londres, 1912, p. 438 (1. a ed. 1889). Sobre el rechazo de la pangénesis $c f$. DARWIN, F. (ed.), The Autobiography of Charles Darwin and Selected letters, Dover, Nueva York, 1958, p. 281.

${ }^{21}$ Se ha demostrado que, al menos, Darwin pudo conocer el trabajo de Mendel mediante el libro de H. Hoffmann, Untersuchungen zur bestimmung des werthes von species und varietät, Gies- 
para lograr la plena aceptación del principio darwinista; él mismo lo anunció en años posteriores ${ }^{22}$. Duda y pregunta son inmediatas, ¿podemos aceptar el testimonio como un reconocimiento implícito de las dificultades añadidas por Mendel a su teoría? Aunque la tentación sea afirmativa objetivamente no es posible asegurarlo y sí atribuir la opinión a su fracaso experimental ${ }^{23}$. El hecho no es relevante, lo importante es el sentido evolutivo marcado por la norma mendeliana, distante del principio selectivo planteada por Darwin. Bajo el peso de la ley genética la especie no evoluciona influenciada por el medio ni está controlada por la selección natural, es en la azarosa combinación de los caracteres parentales dentro del marco exclusivo de la línea celular germinal cuando se generan otros genotipos que darán lugar, fortuita e intermitentemente, a nuevas especies puras ${ }^{24}$. En tal caso, la selección natural ni produce formas nuevas ni su acción es continua y unidireccional, su manifestación se reduce al incremento selectivo del número de individuos con mutaciones favorables frente al resto. Esta concentración poblacional determinará una tendencia morfológica probabilística al ser más verosímil que las futuras modificaciones ocurran en el grupo mayoritario; mero cálculo de probabilidades. Así lo interpreta y expone un mendelista reputado galardonado con el premio Nobel, Thomas Hunt Morgan ${ }^{25}$. Simultáneamente, el ideario mendeliano se postula como alternativa epistemológica realizando un heterodoxo giro metodológico. Dos aspectos a resaltar del nuevo episteme: uno el cambio orientativo cognitivo que supone analizar el problema desde la fisiología celular hasta su realidad fenotípica específica, individualizando la expresión evolutiva desde la inicial fase informativa hasta la conversión final en objeto real permanente; otro integrar la evolución como una manifestación no competitiva, coordinando génesis y consolidación de nuevos sistemas biológicos mediante la reproducción. Evolucionar resulta de la acción armónica de la naturaleza, deriva de la mecánica vital, está ahí pero no es un fin sólo una ten-

sen, 1869. Cf. Galera, A., «Los guisantes mágicos de Darwin y Mendel», Asclepio, vol. LII, fasc. 2, 2000, pp. 214-5.

22 DARWIN, Ch., The effects of cross and selffertilisation in the vegetable kingdom, Murray, Londres, 1876, p. 27.

${ }^{23}$ Cf. Darwin, F. (ed.), The Autobiography of Charles Darwin and Selected letters, Dover, Nueva York, 1958, p. 321.

${ }^{24}$ Un análisis detallado del problema véase en GALERA, A., "Los guisantes mágicos de Darwin y Mendel», Asclepio, vol. LII, fasc. 2, 2000, pp. 213-222.

${ }_{25}$ Morgan, T. H., Evolución y mendelismo. Crítica de la teoría de la evolución, Calpe, Madrid, 1921; cf. pp. 137, 170. 
dencia sostenida por la inconsciente condición mudable del ser vivo. Este principio natural no finalista lo anunció Kant con carácter general en la Crítica del juicio ${ }^{26}$, y lo cumple también la selección darwiniana aunque adornada con una aparente direccionalidad entorno al perfeccionamiento morfológico marcado por la acción de sobrevivir, convertida en diseño anatómico. El diseño es real pero sin intención, la percepción finalista es una virtualidad consecuencia de observar el producto sin haber leído el libro de instrucciones. Se tardaron décadas en percibir y adoptar los cambios derivados del modelo mendeliano pero la revolución genética será el subsiguiente movimiento adaptativo neodarwinista, obligado; un enfoque diferente de la selección natural nominado en última instancia sintesis moderna.

En History of western philosophy del año 1946, el filósofo Bertrand Russell deconstruye la teoría darwinista en sendas partes ya trilladas: una general sobre la conocida doctrina de la evolución, repetida por Darwin donde él interfiere con valor testimonial, aportando pruebas del suceso; otra específica relativa a la selección natural, la innovación, concepto discutible y discutido sujeto a revisión por los biólogos, representativo de «una suerte de economía biológica en un mundo de libre competencia ${ }^{27}$, la consecuencia de aplicar al comportamiento animal y vegetal el esquema socio-económico desarrollado, amplificado, por Thomas Robert Malthus. Traer a colación el dictamen de Russell tiene doble intención, sirve de conciso resumen y sitúa a la selección natural ante un nuevo horizonte: la naturaleza en guerra ${ }^{28}$.

Corría el mes de agosto de 1858, el Journal de la londinense Linnean Society pone negro sobre blanco el académico debate Darwin versus Wallace para dictar sentencia sobre la paternidad de la teoría evolutiva por selección natural ${ }^{29}$. Mera pantomima oficialista porque el resultado no ofrecía dudas. Darwin presentó como alegato un fragmento perteneciente a la versión manuscrita del Origen con-

${ }^{26}$ Kant, I., Critica del juicio, Espasa-Calpe, Madrid, 1977, p. 345.

27 Russel, B., History of western philosophy, Allen \& Unwin, Londres, 1946, pp. 752-753.

28 Magistral y obligatorio es VERGATA, A. La, L'equilibrio e la guerra della natura, Morano, Nápoles, 1990.

29 DARWIN, Ch., Journal of the proceedings of the Linnean society, n. ${ }^{\circ} 9$, agosto, 1858, vol. III, pp. 45-63. Las comunicaciones se presentaron en la sesión del 30 de junio de 1858 por Ch. Lyell y J. D. Hooker. 
cluida en 1844, concretamente el extracto del segundo capítulo titulado On the variation of organic beings in a state of nature; on the natural means of selection; on the comparison of domestic races and true species. Fue la primera manifestación impresa de la teoría. Para la ocasión Darwin no esconde las cartas, al contrario, da un golpe de efecto revelando la autoridad del botánico Augustin-Pyramus De Candolle con la inequívoca intención de reforzar y refrendar su ideario. «De Candolle, en un pasaje elocuente, ha declarado que toda la naturaleza está en guerra, un organismo contra otro o con la naturaleza circundante», es el ideológico párrafo inicial del documento ${ }^{30}$. Astutamente, Darwin encubre la antagónica posición del maestro, concordante en visionar la naturaleza como entidad abierta en permanente competencia; discordante en concluir que tal proceder biológico pervierte los fundamentos de las especies, las transforme; partidario de enfocar el concepto de especie como unidad constante frente a los defensores de su no permanencia real ${ }^{31}$. Géographie botanique es un conocido texto, incluso por Darwin $^{32}$, donde el naturalista suizo expuso su peculiar visión botánica de una naturaleza conflictiva. Resumiendo: todas las plantas de un determinado lugar están en guerra unas contra otras; los primeros ocupantes del espacio tienden a ser excluyentes; las grandes sofocan a las pequeñas; las especies longevas remplazan a aquellas que no lo son; las más fecundas invaden el puesto de las otras ${ }^{33}$. La interacción orgánica no cesa en el teatrum naturae alimentada por un flujo continuo de intereses particulares; conflicto resuelto en forma de distribución geográfica definida por dos variables correlativas: las condiciones de existencia y la organización individual. Las claves del proceso evolutivo-competitivo aparecen aquí en su versión vegetal, conocimiento que Darwin maceró junto al malthusiano principio de supervivencia. Triangulación ideológica, lucha $\leftrightarrow$ demografía $\leftrightarrow$ selección (Candolle $\leftrightarrow$ Malthus $\leftrightarrow$ Darwin), significativamente evidente ya en el borrador ${ }^{34}$ del Origen redactado en 1842 . La guerra natural obtuvo una

30 Ibídem, p. 46.

31 Candolle, A. P. De, Théorie élémentaire de la botanique, ou Exposition du principe de la classification naturelle et de l'art de décrire et d'étudier les végétaux, Déterville, París, 2.a ed., 1919, pp. 195-6.

32 Darwin estudio en la universidad de Edimburgo la teoría botánico de A. P. De Candolle y se entrevisto con él en 1839. Sobre el tema, por ejemplo, $c f$. DESMOND, A.; MOORE, J. (1991), Darwin, Penguin, Londres, 1991, pp.43, 265, 283.

33 Candolle, A. P. De, Géographie botanique, Levrault, París, 1820, p. 26.

${ }^{4}$ Citamos por DARWIN, F. (ed.), The foundations of the origins of species. Two essays written in 1842 and 1844, University press, Cambridge, 1909, p.7; cf. también las pp. 87-88 del ms. redactado en 1844. 
expresión superlativa con la forma y el sentido darwiniano, dejando de ser el antecedente concepto fijista regulador de una naturaleza inalterable gobernada por el principio de crecer y multiplicarse que tampoco eximía de temporalizar el hecho orgánico-formativo, secuenciación imprescindible para respetar la bipolarización autotrófica-heterotrófica de los organismos; sí, será una cronología muy reducida suficiente para reproducirse sin presión demográfica. Al contemplar los objetos como alimento el orden natural se desvía hacia una gradación trófica ascendente desde las plantas hasta el hombre; servidumbre nutricional que obliga a establecer un período genésico distinto entre niveles, tiempo necesario para aumentar suficientemente el número de individuos evitando que las especies desaparezcan convertidas en nutrientes.

El cuento lo aplicó el célebre Linneo, y Kant tomó nota dando un paso hacia delante. Al relacionarlos nutricionalmente, los objetos vivos definen una naturaleza finalista existencial: cada agrupación trófica permite la subsistencia de otros, sin embargo, tal y como razona el filósofo ${ }^{35}$, el camino puede recorrerse en otro sentido con distinto resultado. De arriba hacia abajo, convirtiendo a los grupos en unidades de control poblacional, de suerte que el anterior fin se convierte en medio de conseguir algo diferente. Cada elemento es simultáneamente sujeto y objeto de la acción, proceso reglado mediante un principio de necesidad, energético, conducente al equilibrio sistémico por retroalimentación. Regido por la selección natural el modelo mantiene su esencia porque, en definitiva, la norma darwinista no deja de ser una refinada aplicación matemática de la antecedente cadena trófica, pasando de la descripción linneana a la cuantificación malthusiana. La guerra natural selectiva es una lucha por el alimento con repercusiones morfológicas, alterando una naturaleza donde se ha perdido la constancia específica que subyugaba el sistema por mandato divino. Pero, ¿realmente la selección natural supone una renuncia a la figura del creador?; o como reza la proposición kantiana: «¿hay a la base de lo que, con razón, llamamos fin de la naturaleza, un ser que obra intencionadamente como causa del mundo (por tanto como creador)? $\aleph^{36}$.

Cuando el año 1831 embarca en el Beagle, Darwin es un confeso teísta fijista partidario de la teoría del diseño esgrimida por William Paley, prueba con-

\footnotetext{
35 Kant, I., Crítica del juicio, Espasa-Calpe, Madrid, 1977, p. 343.

36 Ibídem, p. 314.
} 
cluyente de la creación y el determinismo de la naturaleza ${ }^{37}$. La evolución era un pensamiento fuera de lugar. El autor del Origen confiesa que hasta 1839 no se persuade de la mutabilidad y la descendencia común de los organismos ${ }^{38}$, aunque su nuevo ideario no refuta, necesariamente, la existencia de un ser supremo. En la escenografía darwinista el plan de Dios no es generar seres vivos, la creación se reduce a un suceso antiguo restringido a la aparición de materia y las consiguientes leyes generales que la gobiernen ${ }^{39}$. La vida será un hecho probabilístico a desarrollarse partiendo de esta primaria organización. Se rescribe la historia, y ¿cuál es el papel de Dios en la evolución? Respondiendo, Darwin utiliza el símil de la selección artificial pues no en vano la Biblia señala el camino ${ }^{40}$. El origen de los organismos se reduce a la creación primigenia de elementales formas vivas, materia dotada de capacidad sensorial, crecimiento y multiplicación ${ }^{41}$, desde entonces el creador asemeja a los agricultores y ganaderos, combinando y seleccionando individuos mediante la reproducción ${ }^{42}$. Actúa indirectamente a través de la selección natural, convertida en la mano alargada de Dios que mece la cuna de la naturaleza transformándola. La selección natural es la ley general que, con su consentimiento y sin su intervención, gobierna las relaciones individuo-medio componiendo una biogénesis divina a distancia. Complementando dicha interpretación, el modelo transformista darwiniano corrobora un estatus de perfección morfológica que direcciona y restringe la variabilidad real ${ }^{43}$; esquema donde la lucha por la supervivencia es el filtro para elegir al más apto, posibilita la sustitución de una especie por otra y causa la extinción. Los tres pilares del catecismo evolutivo darwinista. Todo ocurre como un incesante juego combinatorio donde los seres vivos proliferan y desaparecen mientras el planeta gira; hay "grandeza en esta concepción de que la vida, con sus diferentes fuerzas, ha sido alen-

37 Cf. Francis Darwin (ed.), The autobiography of Charles Darwin and selected letters, Dover, Nueva York, 1958, pp. 63, 66, 175 y 184. Darwin estudio en Cambridge las obras de Paley; ibídem, p. 19.

38 Ibídem, p. 175.

39 DARWIN, F. (ed.), The foundations of the origins of species. Two essays written in 1842 and 1844, University press, Cambridge, 1909, pp. 51-52, 254.

${ }^{40}$ Ibídem, p. 67.

41 DARWIN, Ch., The origin of species, Murray, Londres, 1876, 6 ${ }^{\mathrm{a}}$ ed. p. 429 (1. ${ }^{\mathrm{a}} \mathrm{ed}, 1859$, p. 490).

42 DARWIN, F. (ed.), The foundations of the origins of species. Two essays written in 1842 and 1844, University press, Cambridge, 1909, pp. 6-7, 83-85.

43 Ibídem, pp. 23,134. 
tada por el Creador», suscribe Darwin al final de la sexta edición del Origen ${ }^{44}$. Queremos imaginar que Darwin leyó a Kant aprendiendo de él que sólo un ser inteligente puede encabezar los supuestos fines de la naturaleza; teleología que, sin embargo, no prueba la existencia de dicha entidad teológica y debe interpretarse como un principio interno derivado de causas naturales. La aceptación figurante es consecuencia de un fundamento subjetivo inherente e indispensable para el hombre ${ }^{45}$. Si Darwin careció de la necesaria lectura la convergencia ideológica es coincidencia; sea como fuere, Dios existe en su modelo evolutivo primero en calidad de proveedor, el resto como único espectador de un combate por la vida guiado por la selección en pos del progreso y la perfección.

Para concluir recurrimos a la metáfora darwiniana de una selección natural que, silente e imperceptiblemente, busca, día y noche, por tierra mar y aire, minuto a minuto, cualquier variación rechazando las perjudiciales e incorporando las beneficiosas, perfeccionando cada ser en relación con sus condiciones de vida ${ }^{46}$. Una definición inequívoca de la cualidad omnímoda de una selección que como Dios lo abarca todo.

\section{Bibliografía}

BOHR, N.,1999. I quanti e la vita, unità de la natura unità della conoscenza, Bollati, Turín.

Candolle, A. P. De, 1820. Géographie botanique, Levrault, París.

- 1919. Théorie élémentaire de la botanique, ou Exposition du principe de la classification naturelle et de l'art de décrire et d'étudier les végétaux, Déterville, París, 2. ${ }^{a}$ ed.

- 1862. «Etude sur l'espèce à l'occasion d'une révision de la famille des cupuliferes», Annales des sciences naturelles, $4 .^{\mathrm{a}}$ serie, Botanique, t. XVIII.

DARWIN, Ch., 1858. Journal of the proceedings of the Linnean society, n. ${ }^{\circ}$ 9, agosto, vol. III, pp. 45-63.

- 1859. On the origin of species by means of natural selection, John Murray, Londres.

44 DARWIN, Ch., The origin of species, Murray, Londres, 1876, 6. a ed. p. 429 (1. a ed, 1859, p. 490).

45 Kant, I., Crítica del juicio, Espasa-Calpe, Madrid, 1977, pp. 293, 314.

46 Darwin, Ch., The origin of species, Murray, Londres, 1876, pp. 65-66. 
- 1868. The variation of plants and aimals under domestication, John Murray, Londres.

- 1876. The effects of cross and self fertilisation in the vegetable kingdom, Murray, Londres.

DARWIN, F. (ed.), 1909. The foundations of the origins of species. Two essays written in 1842 and 1844, University press, Cambridge.

- 1958. The Autobiography of Charles Darwin and Selected letters, Dover, Nueva York.

Desmond, A.; Moore, J., 1991. Darwin, Penguin, Londres.

Drouin, J-M.; Lenay, CH. (eds.), 1990. Théories de l'évolution, une anthologie, Pocket, París.

FISHER, R. A., 1958. The genetical theory of natural selection, Dover, Nueva York.

GALERA, A., 2009. «Lamarck y la conservación adaptativa de la vida», Asclepio, vol. LXI, 2, pp. 129-140.

- 2000. «Los guisantes mágicos de Darwin y Mendel», Asclepio, vol. LII, fasc. 2, pp. 214-215.

— 2002. «Modelos evolutivos predarwinistas», Arbor, 172 (677), pp. 1-16.

GÉRARD, F.,1844/5. Dictionnaire Universel d'Histoire Naturelle: «Dégénérescence», 1844, t. 4, pp. 647-655; «Espèce», 1844, t. 5, pp. 428-452; «Géographie zoologique», 1845, t. 6, pp. 112-192; "Generation spontaneée ou primitive», 1845, t. 6, pp. 53-71.

Guedes, E.(ed.), 2006. Numeros e outras coisas da vida, Apenas livros, Lisboa.

Huxley, J., 1943. Evolution The Modern Synthesis, G. Allen \& Unwin Ltd., Londres.

KanT, I., 1977. Crítica del juicio, Espasa-Calpe, Madrid.

Lamarck, J. B., 1994. Philosophie zoologique, Flammarion, París.

LaUrent, G., 2001. La naissance du transformismo. Lamarck entre Linné et Darwin, Vuibert, París.

Morgan, T. H., 1921. Evolución y mendelismo. Critica de la teoría de la evolución, Calpe, Madrid.

Quatrefages, A. De, 1892. Charles Darwin et ses précurseurs françaises, París.

Russel, B., 1946. History of western philosophy, Allen \& Unwin, Londres.

Simpson, G. G., 1967. La vida en el pasado, Madrid, Alianza..

Vergata, A. La, 1990. L'equilibrio e la guerra della natura, Morano, Nápoles. 
Wallace, A. R., 1912. Darwinism. An exposition of the theory of natural selection with some of its applications, Mcmillan, Londres.

Recibido:10/10/2009

Revisado: 20/10/2009 